\title{
Effect of Vitamin D3 Supplementation Combined with Exercise Training on Glycemic Control and Bone Health in Patients with Type 2 Diabetes: A Randomized, Placebo-Controlled Trial
}

Xiaomin Sun ( $\nabla$ gzhtxiaomin@163.com )

Xi'an Jiaotong University https://orcid.org/0000-0002-6473-6311

Sirui Zhou

Xi'an Jiaotong University

Mengyue Dong

Xian Physical Education University

Wenjuan Xiao

Xi'an Jiaotong University

Xin He

Xi'an aerospace general hospital

Yan Ma

Xian Physical Education University

Bo Gou

Xian Physical Education University

Jing Xu

Second Affiliated Hospital of xi'an jiaotong university

\section{Zhenbo Cao}

Shanghai University of Sport

Lingxia Zeng

Xi'an Jiaotong University

Youfa Wang

Xi'an Jiaotong University

Wei Cui

first affiliated hospital of Xi'an Jiaotong University

\section{Research article}

Keywords: bone mass, diabetes, exercise training, glycemic control, vitamin D supplementation

Posted Date: December 31st, 2020 
DOI: https://doi.org/10.21203/rs.3.rs-137382/v1

License: (c) (1) This work is licensed under a Creative Commons Attribution 4.0 International License. Read Full License 


\section{Abstract}

\section{Aims}

This study aimed to examine the effect of a 12-week vitamin D supplementation and exercise training alone and in combination on glycemic control and bone health in Chinese type 2 diabetes patients.

\section{Methods}

Sixty-one type 2 diabetes patients (age, 33-65 years; $72.0 \%$ men) with non-insulin dependence were randomized into the 12-week vitamin D group (1000 IU/day), exercise group (60\%-80\% of maximal heart rate, $1 \mathrm{~h} /$ time, 2-3 times/week), vitamin D combined with exercise group, and control group. A 75-g oral glucose tolerance test was used to estimate glycemic control. Dual X-ray absorptiometry was used to examine bone health (bone mass content and bone mass density) and body fat percentage (\%).

\section{Results}

During the 75-g oral glucose tolerance test, lower glucose and higher insulin levels were found in the vitamin D combined with exercise group, vitamin D group, and exercise group after intervention than before intervention, although the differences were not statistically significant. A significant exercise and vitamin $D$ interaction for the insulinogenic index $(P=0.032)$ and a borderline interaction for the glucose disposition index $(P=0.051)$ were observed, while no further independent effect was observed. Compared with non-vitamin $\mathrm{D}$ supplementation, vitamin $\mathrm{D}$ supplementation significantly alleviated the loss of total bone mass content (95\% Cl: $-29.9-19.4$ vs. $-74.9--24.7)$, trunk bone mass content ( $95 \% \mathrm{Cl}$ : $-24.1-19.5$ vs. $-56.1--11.7)$, and spine bone mass density (95\% Cl: $-0.03-0.03$ vs. $-0.07--0.01)$.

\section{Conclusions}

The findings suggest that 12-week combined vitamin $D$ and exercise intervention has a potentially positive effect on glycemic control, and vitamin D supplementation plays an important role in the prevention of bone loss, which was identified in the exercise alone group. Further studies are needed to elucidate the long-term effect of combined vitamin D and exercise intervention in type 2 diabetes patients.

The study was registered in the Chinese Clinical Trial System (No. ChiCTR1800015383).

\section{Introduction}

With rapid social and economic developments during the past three decades, China is facing a growing threat from non-communicable chronic diseases (NCDs), and diabetes is considered one of the most common NCDs in China and several other countries ${ }^{1}$. Diabetes prevalence in Chinese adults increased substantially from $0.67 \%$ in 1980 to $9.7 \%$ in 2008 and $10.9 \%$ in $2013^{2,3}$. Studies have demonstrated that intentional weight loss and increased physical activity intervention are associated with high rates of bone 
loss in patients with type 2 diabetes ${ }^{4}$. Thus, optimizing type 2 diabetes management without a negative effect on bone mass has become one of the emphases in the treatment of type 2 diabetes.

Accumulating evidence indicates that high 25-hydroxycholecalciferol $(25(\mathrm{OH}) \mathrm{D})$ levels are positively associated with areal bone mineral density (BMD). Accordingly, vitamin D supplements are widely recommended and prescribed in the general population to promote bone health ${ }^{5}$. In addition, altered vitamin $\mathrm{D}$ homeostasis may play a role in the development of insulin resistance (IR) and type 2 diabetes 6 , and low 25(OH)D levels are associated with a high prevalence of type 2 diabetes ${ }^{7,8}$. Therefore, vitamin D supplementation has been proposed as a potential intervention to lower the incidence of type 2 diabetes ${ }^{9,10}$.

Vitamin $\mathrm{D}$ deficiency $(25(\mathrm{OH}) \mathrm{D}<20 \mathrm{ng} / \mathrm{mL})$ is prevalent in several populations and has become a common public health problem worldwide due to sun protection measures, reduction of outdoor activities, and environmental pollution ${ }^{5}$. Zhang et al. recently showed that in China, $50 \%$ of type 2 diabetes patients were vitamin $D$ deficient, which could further deteriorate glucose tolerance status ${ }^{11}$. However, to date, studies on the role of vitamin D supplementation in IR in type 2 diabetes patients have been mainly conducted in non-Asian countries, and the findings are inconsistent ${ }^{12-17}$, partly because of marked differences in study duration, participant population, and dosing regimen ${ }^{9,10}$. Furthermore, the independent effect of vitamin D on glycemic control is possibly limited in type 2 diabetes patients ${ }^{18}$. For example, Krul-Poel et al. found that 6-month vitamin D supplementation had no effect on HbA1c and fasting glucose levels and IR in type 2 diabetes patients ${ }^{12}$, and similar results were also observed in German and Chinese type 2 diabetes patients ${ }^{13,19}$. Therefore, further studies are needed to clarify the role of vitamin D supplementation in type 2 diabetes patients.

Exercise intervention is one of the most effective traditional lifestyle interventions to prevent and treat type 2 diabetes ${ }^{20}$. Recent studies have shown that exercise may be involved in the regulation of vitamin $D$ and increases vitamin $D$ receptor (VDR) expression in skeletal muscle and serum 25(OH)D levels ${ }^{21-23}$. Moreover, in addition to regulating $\mathrm{Ca}^{2+}$ levels, vitamin $\mathrm{D}$ supplementation can directly upregulate the AMP-activated protein kinase (AMPK)-glucose transporter 4 (GLUT-4) signaling pathway through VDR to promote exercise-associated glucose utilization ${ }^{24}$. These results suggest that vitamin $\mathrm{D}$ supplementation combined with exercise intervention might have a synergistic effect on the improvement of glycemic control by activating different glucose utilization pathways. However, few studies have investigated the synergistic effect of vitamin D supplementation and exercise training intervention on glycemic control in type 2 diabetes patients.

This study aimed to examine the effects of 12-week vitamin D supplementation combined with exercise training on glycemic control, as well as bone health in Chinese type 2 diabetes patients. Our findings will provide a comprehensive understanding of combined vitamin $D$ and exercise intervention on glycemic control in Chinese type 2 diabetes patients. 


\section{Methods}

\section{Experimental design}

This 12-week $2 \times 2$ factorial design, randomized, placebo-controlled trial assessed the impact of combined vitamin $D$ and exercise intervention on glycemic improvement in type 2 diabetes patients with non-insulin dependence in Xi'an, China ( $34^{\circ} \mathrm{N}$ latitude). The purpose, procedures, and risks of the study were explained to each participant before inclusion, and all participants provided written informed consent. All of the procedures were reviewed and approved by the Ethics Committee of Xi'an Jiaotong University Health Science Center. The study was conducted in accordance with the Declaration of Helsinki and registered in the Chinese Clinical Trial System (No. ChiCTR1800015383). The complete process is outlined in Fig. 1.

<Figure 1 about here>

An investigator who was blinded to this study randomly divided the participants into the following four groups based on a computer-generated random allocation table stratified according to age and sex: vitamin $D$ combined with exercise training group (VEG), vitamin D supplementation group (VDG), exercise group (EG), and placebo control group (CG). To ensure the randomized, double-blind effect, trial designers, testers, and data collectors were blinded to the vitamin D/placebo intake grouping until the intervention trial and data collection were completed. All patients were instructed not to undertake any formal exercise or change their general physical activity levels and dietary habits during the intervention period. Participants were asked to abstain from caffeine, alcohol, tobacco, and strenuous physical activity before the day of blood sample collection. All measurements were assessed at baseline and at the end of the intervention.

\section{Participants}

Sixty-one type 2 diabetes patients aged 33-65 years without receiving insulin treatment were enrolled between 2017 and 2018. Patients were eligible for study participation if they met the following inclusion criteria: (a) diagnosed with type 2 diabetes according to the WHO (1999) ${ }^{25}$ and had type 2 diabetes for $\leq$ 10 years, (b) no plan to replace the hypoglycemic agent in the near future, (c) without regular vitamin $D$ and/or calcium supplements in the past year, (d) did not meet the current National Physical Activity guidelines, and (e) without regular exercise habits in the past year.

The following participants were excluded: participants with acute infection; participants experiencing stress or acute complications of diabetes; participants with heart, liver, and kidney insufficiency, osteoporosis and fracture, and metal implants in the body that could affect magnetic resonance imaging and dual-energy X-ray absorptiometry (DXA) measurements; and participants who had used insulin therapy and had a recent history of sunlight exposure. 


\section{Interventions}

\section{Vitamin D intervention (double-blind)}

Patients in the VDG or VEG group received one tablet of vitamin $D_{3}$ supplement (1000 IU/day, Nature Made, Otsuka Pharmaceutical Co, Ltd, Tokyo, Japan), and those in the EG or CG group received a placebo tablet every day for 3 months. The tablets of vitamin $D_{3}$ supplements and placebos had identical appearance, shape, and color. Placebo tablets contained only starch, cellulose, and magnesium stearate. All supplements were prepared in identical bottles and sent to their homes or hand-delivered monthly. Participants were asked to report remotely their supplements use weekly.

\section{Exercise intervention}

Patients in the EG or VEG group performed a 1-h progressively increasing aerobic exercise (cycling, running, or rowing) at $60-80 \%$ of maximal heart rate $\left(\mathrm{HR}_{\max }\right) 2-3$ times a week for 12 weeks. This was supervised by a qualified trainer who is knowledgeable of the study protocol and procedures. A polar monitor was used to monitor heart rate during exercise, and compliance with each protocol was recorded. Participants warmed up during the first 5 min on a treadmill at $50-60 \%$ of $\mathrm{HR}_{\max }$ and subsequently followed the exercise protocol assigned to them, with a 5-10-min recovery exercise at $40-50 \%$ of $\mathrm{HR}_{\max }$ which comprised walking and stretching exercises.

\section{Primary Outcomes}

\section{Glycemic control parameters}

A standard 75-g OGTT was performed between 08:30 and 11:00 after a 12-h overnight fast, and venous blood samples were collected in Venoject-II AutoSep tubes at 0, 30, 60, 90, and 120 min to determine plasma glucose and serum insulin levels. Serum samples were used to measure 25(OH)D, fasting glucose $\left(\mathrm{G}_{0}\right)$, fasting insulin $\left(\mathrm{I}_{0}\right), \mathrm{HbA} 1 \mathrm{c}$, triglyceride, cholesterol, low-density lipoprotein cholesterol, highdensity lipoprotein cholesterol, apolipoprotein (Apo)A-1, and ApoB levels. Fasting blood glucose and insulin levels were used to calculate the insulin resistance index (homeostatic model assessment of insulin resistance [HOMA-IR]) as follows: $\mathrm{HOMA}-\mathrm{IR}=\mathrm{G}_{0} \times \mathrm{I}_{0} / 22.5$.

Insulin sensitivity estimated using the Matsuda index during the OGTT was calculated as follows: 1000 /square root of $\left(\left[G_{0} \times I_{0}\right]\right) \times($ mean OGTT glucose concentration $\times$ mean OGTT insulin concentration) 26 . The early phase of insulin secretion was estimated using the insulinogenic index (IGI) as follows: $\mathrm{I}_{30^{-}}$ $\mathrm{I}_{0} / \mathrm{G}_{30}-\mathrm{G}_{0}(\triangle \mathrm{I} 30 / \triangle \mathrm{G} 30)$, where $\mathrm{I}_{30}$ and $\mathrm{G}_{30}$ represent insulin and glucose values at 30 min during the OGTT, respectively ${ }^{27}$. The glucose disposition index (GDI) was calculated based on insulin secretion and insulin resistance as follows: Matsuda index $\times I G I$. The increments in the area under the curves during the 
complete 120-min period of the OGTT were calculated using the trapezoid rule to assess the total changes in glucose and insulin levels.

\section{Secondary Outcomes \\ Body fat and bone mass}

Height and body mass were measured with the participants wearing light clothing and barefoot. Body mass index was calculated by dividing the body mass in kilograms by the square of height in meters $\left(\mathrm{kg} / \mathrm{m}^{2}\right)$. Waist circumference was measured to the nearest $0.1 \mathrm{~cm}$ at the umbilical region using an inelastic measuring tape at the end of normal expiration. DXA was used to measure body fat percentage (\%) and bone mass (bone mass content [BMC] and BMD) (Hologic QDR-4500, DXA Scanner, Hologic Inc., Waltham, MA, USA) by a recognized technologist.

\section{Sunlight exposure}

Participants were instructed to record their outdoor activity time and exposed areas of the skin from 9 am to $5 \mathrm{pm}$ for 7 consecutive days in a week using a questionnaire before and after the intervention. A score to estimate the mean weekly sunlight exposure was calculated, and additional details were published elsewhere ${ }^{28}$.

\section{International Physical Activity Questionnaire}

Daily physical activity, except for the exercise program, was assessed using the International Physical Activity Questionnaire monthly and expressed as metabolic equivalent minutes per week. This questionnaire refers to activities in the preceding 7 days. Participants were instructed to report the number of days, hours, and minutes spent on vigorous activities, including aerobics, or moderate activities, such as carrying light loads ${ }^{29}$.

\section{Statistical Analyses}

\section{Power analysis}

Based on a previous study examining the association of vitamin D intervention with HOMA-IR ${ }^{30}$, the sample size required for each group was estimated to be 12 with a power of $85 \%$, and an effect size of 0.26 was assumed. Therefore, 48 type 2 diabetes patients were required. To account for $20 \%$ loss to follow-up, 60 type 2 diabetes patients were recruited. Power calculations were performed using G*Power software version 3.1.9.2 ${ }^{31}$.

\section{Data analysis}


Descriptive statistics were calculated using means (SDs) for continuous variables, $n$ (\%) for categorical variables, or $95 \% \mathrm{Cl}$ unless otherwise stated. Differences at baseline were compared using one-way ANOVA for continuous variables and chi-squared tests for categorical variables. Repeated measures ANOVA was used to analyze the effect of vitamin D supplementation on serum $25(\mathrm{OH}) \mathrm{D}$ levels. A $2 \times 2$ factorial design was used to estimate the effects of vitamin $D$ and exercise intervention on metabolic profiles and bone health. A post hoc test with Bonferroni correction was used to identify significant differences among the mean values when a significant main effect or interaction was identified. All data were analyzed based on the intention to treat. Statistical significance was set at $P<0.05$.

\section{Results}

\section{Baseline characteristics}

Sixty-one eligible participants (44 men and 17 women) underwent initial assessments and subsequent randomization (Fig. 1). During the intervention, one participant in the VDG and another in the VEG dropped out for personal reasons, and all participants in the CG and EG completed the intervention. In addition, one participant in the VDG and another in the CG did not complete DXA measurement, and two participants in the VDG and the other two participants in the CG did not complete the OGTT. Of the 29 participants who were allocated to the exercise groups, 20 participants completed 2-3 times exercise per week, and three completed 20 times exercise during the intervention period.

Baseline characteristics of participants are detailed in Table 1. The study participants had a mean BMI of $25.8 \pm 3.6 \mathrm{~kg} / \mathrm{m}^{2}$ and a mean age of $50.0 \pm 7.6$ years at baseline. There were no significant differences between the four groups in age, men\%, BMl, body fat percentage, sun exposure score, physical activity, and glucose profiles at baseline (Table 1). In addition, of the 61 participants, $40(65.6 \%)$ had vitamin D deficiency $(25(\mathrm{OH})$ D levels $<20 \mathrm{ng} / \mathrm{mL})$, and 19 (31.1\%) had vitamin $\mathrm{D}$ insufficient $(25(\mathrm{OH}) \mathrm{D}$ levels: $20-$ $30 \mathrm{ng} / \mathrm{mL})$. 
Table 1

Subject characteristics at baseline

\begin{tabular}{|c|c|c|c|c|c|c|}
\hline \multirow[t]{2}{*}{ Variable } & Overall & VEG & VDG & EG & CG & \multirow[t]{2}{*}{$P$} \\
\hline & $n=61$ & $n=16$ & $n=16$ & $n=14$ & $n=15$ & \\
\hline Age (years) & $50.0 \pm 7.6$ & $51.3 \pm 6.9$ & $50.1 \pm 8.3$ & $47.9 \pm 8.6$ & $50.7 \pm 7.0$ & 0.763 \\
\hline Male (\%) & $\begin{array}{l}44 \\
(72.0 \%)\end{array}$ & $\begin{array}{l}11 \\
(64.7 \%)\end{array}$ & $\begin{array}{l}11 \\
(73.3 \%)\end{array}$ & $\begin{array}{l}11 \\
(73.3 \%)\end{array}$ & $\begin{array}{l}11 \\
(78.6 \%)\end{array}$ & 0.855 \\
\hline Height (cm) & $\begin{array}{l}167.5 \pm \\
7.5\end{array}$ & $\begin{array}{l}165.4 \pm \\
7.9\end{array}$ & $\begin{array}{l}167.7 \pm \\
8.2\end{array}$ & $\begin{array}{l}169.1 \pm \\
7.7\end{array}$ & $\begin{array}{l}167.8 \pm \\
6.1\end{array}$ & 0.581 \\
\hline Weight (kg) & $\begin{array}{l}72.7 \pm \\
13.0\end{array}$ & $\begin{array}{l}68.6 \pm \\
12.7\end{array}$ & $\begin{array}{l}71.3 \pm \\
12.7\end{array}$ & $\begin{array}{l}74.7 \pm \\
15.1\end{array}$ & $\begin{array}{l}76.9 \pm \\
10.9\end{array}$ & 0.316 \\
\hline $\mathrm{BMI}\left(\mathrm{kg} / \mathrm{m}^{2}\right)$ & $25.8 \pm 3.6$ & $24.9 \pm 3.0$ & $25.3 \pm 3.2$ & $26.1 \pm 4.8$ & $27.2 \pm 3.2$ & 0.279 \\
\hline Body fat (\%) ${ }^{a}$ & $30.1 \pm 7.6$ & $31.6 \pm 6.0$ & $27.6 \pm 8.9$ & $30.9 \pm 8.6$ & $30.3 \pm 6.6$ & 0.640 \\
\hline Trunk body fat (\%) a & $36.5 \pm 7.8$ & $37.4 \pm 6.7$ & $33.9 \pm 9.3$ & $37.7 \pm 8.2$ & $37.3 \pm 6.8$ & 0.652 \\
\hline $\begin{array}{l}\text { Physical activity (MET- } \\
\text { min/week) }\end{array}$ & $\begin{array}{l}3633 \pm \\
3432\end{array}$ & $\begin{array}{l}3161 \pm \\
2331\end{array}$ & $\begin{array}{l}4272 \pm \\
4504\end{array}$ & $\begin{array}{l}4334 \pm \\
4286\end{array}$ & $\begin{array}{l}2801 \pm \\
1976\end{array}$ & 0.788 \\
\hline Sun exposure score & $14.9 \pm 7.5$ & $17.3 \pm 8.6$ & $13.1 \pm 4.4$ & $16.1 \pm 8.8$ & $13.2 \pm 7.3$ & 0.561 \\
\hline Diabetes duration (years) & $3.6 \pm 2.6$ & $3.7 \pm 2.9$ & $3.7 \pm 2.4$ & $3.1 \pm 2.5$ & $3.7 \pm 2.6$ & 0.969 \\
\hline HbA1c (\%) & $7.0 \pm 1.5$ & $7.0 \pm 1.8$ & $6.8 \pm 1.4$ & $6.7 \pm 1.1$ & $7.2 \pm 1.6$ & 0.799 \\
\hline $\begin{array}{l}\text { Fasting blood glucose } \\
(\mathrm{mmol} / \mathrm{L})\end{array}$ & $7.4 \pm 2.0$ & $6.8 \pm 1.4$ & $7.4 \pm 1.8$ & $7.8 \pm 2.1$ & $7.5 \pm 2.6$ & 0.626 \\
\hline Fasting insulin $(\mu \mathrm{U} / \mathrm{mL})$ & $12.0 \pm 7.1$ & $12.1 \pm 8.5$ & $9.6 \pm 5.5$ & $11.7 \pm 6.1$ & $4.7 \pm 7.6$ & 0.452 \\
\hline HOMA-IR & $4.0 \pm 2.6$ & $3.8 \pm 2.9$ & $3.4 \pm 2.5$ & $3.9 \pm 2.3$ & $4.9 \pm 2.6$ & 0.598 \\
\hline \multicolumn{7}{|c|}{ 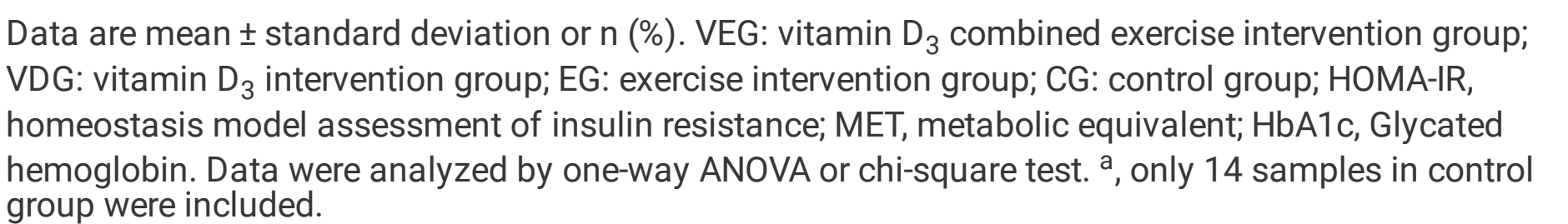 } \\
\hline
\end{tabular}

\section{Serum 25(oh)d Concentrations And Glycemic Control}

After 12-week vitamin D supplementation, serum 25(OH)D levels were increased in the mid and at end of the intervention in the VEG (baseline, $18.1 \pm 2.0 \mathrm{ng} / \mathrm{mL}$; mid, $24.7 \pm 2.1 \mathrm{ng} / \mathrm{mL}$; end, $27.8 \pm 2.4 \mathrm{ng} / \mathrm{mL}$ ) and VDG (baseline, $18.2 \pm 2.4 \mathrm{ng} / \mathrm{mL}$; mid, $22.8 \pm 2.1 \mathrm{ng} / \mathrm{mL}$; end, $25.9 \pm 2.4 \mathrm{ng} / \mathrm{mL}$ ). However, no changes in serum 25(OH)D levels were observed in the EG and CG at any time point (Fig. 2). 
A significant exercise and vitamin $D$ interaction for the IGI $(P=0.032)$ and a borderline significant interaction for $\mathrm{GDI}(\mathrm{P}=0.051)$ were observed. Post hoc analysis showed that in the non-vitamin $\mathrm{D}$ supplementation groups, exercise increased the IGI compared with without exercise, although the difference was not statistically significant (EG: mean $=0.09,95 \% \mathrm{Cl}=-0.00-0.19$; CG: mean $=-0.02,95 \%$ $\mathrm{Cl}=-0.12-0.06 ; \mathrm{P}=0.09)$. In addition, no independent effect on $\mathrm{GDI}$ was observed in any group. There was no significant exercise and vitamin $D$ interaction or any main effects for other blood profiles. Notably, exercise significantly decreased triglyceride levels (main effect: mean $=-0.14,95 \% \mathrm{Cl}=0.00-0.59$ ) (Table 2). 
Table 2

Mean differences $(95 \% \mathrm{Cl})$ of blood parameter from the endpoint with each group

\begin{tabular}{|c|c|c|c|c|c|c|c|}
\hline \multirow[t]{2}{*}{ variable } & \multirow{2}{*}{$\begin{array}{l}\text { VEG } \\
\mathrm{N}=15\end{array}$} & \multirow{2}{*}{$\begin{array}{l}\text { VDG } \\
\mathrm{N}=15\end{array}$} & \multirow{2}{*}{$\begin{array}{l}E G \\
N=14\end{array}$} & \multirow{2}{*}{$\begin{array}{l}\text { CG } \\
N=15\end{array}$} & \multicolumn{3}{|l|}{$P$} \\
\hline & & & & & $\begin{array}{l}P_{\text {vitamin }} \\
\text { D }\end{array}$ & $P_{\text {exercise }}$ & $P_{\text {interaction }}$ \\
\hline $\begin{array}{l}\Delta \\
\text { Apolipoprotein } \\
\mathrm{A}(\mathrm{g} / \mathrm{L})\end{array}$ & $\begin{array}{l}0.02 \\
(-0.1 \\
0.15)\end{array}$ & $\begin{array}{l}0.04 \\
(-0.07 \\
0.15)\end{array}$ & $\begin{array}{l}0.01 \\
(-0.06 \\
0.07)\end{array}$ & $\begin{array}{l}0.08 \\
(-0.05 \\
0.21)\end{array}$ & 0.582 & 0.349 & 0.400 \\
\hline $\begin{array}{l}\Delta \\
\text { Apolipoprotein } \\
\mathrm{B}(\mathrm{g} / \mathrm{L})\end{array}$ & $\begin{array}{l}0.02 \\
(-0.07 \\
0.12)\end{array}$ & $\begin{array}{l}0.03 \\
(-0.03 \\
0.08)\end{array}$ & $\begin{array}{l}0.03 \\
(-0.06 \\
0.12)\end{array}$ & $\begin{array}{l}0.07 \\
(-0.02 \\
0.15)\end{array}$ & 0.232 & 0.986 & 0.327 \\
\hline $\begin{array}{l}\Delta \text { Low density } \\
\text { lipoprotein } \\
(\mathrm{mmol} / \mathrm{L})\end{array}$ & $\begin{array}{l}-0.09 \\
(-0.43 \\
0.25)\end{array}$ & $\begin{array}{l}-0.1 \\
(-0.26 \\
0.06)\end{array}$ & $\begin{array}{l}-0.26 \\
(-0.54 \\
0.01)\end{array}$ & $\begin{array}{l}-0.1 \\
(-0.27 \\
0.06)\end{array}$ & 0.969 & 0.977 & 0.203 \\
\hline $\begin{array}{l}\Delta \text { High density } \\
\text { lipoprotein } \\
\text { (mmol/L) }\end{array}$ & $\begin{array}{l}0.06 \\
(-0.05 \\
0.17)\end{array}$ & $\begin{array}{l}0(-0.16, \\
0.17)\end{array}$ & $\begin{array}{l}0.01 \\
(-0.12 \\
0.15)\end{array}$ & $\begin{array}{l}-0.03 \\
(-0.14 \\
0.07)\end{array}$ & 0.563 & 0.455 & 0.777 \\
\hline $\begin{array}{l}\Delta \text { Cholesterol } \\
(\mathrm{mmol} / \mathrm{L})\end{array}$ & $\begin{array}{l}0.06 \\
(-0.39 \\
0.51)\end{array}$ & $\begin{array}{l}0(-0.29 \\
0.29)\end{array}$ & $\begin{array}{l}-0.19 \\
(-0.5 \\
0.12)\end{array}$ & $\begin{array}{l}-0.11 \\
(-0.37 \\
0.14)\end{array}$ & 0.699 & 0.691 & 0.281 \\
\hline $\begin{array}{l}\Delta \text { Triglyceride } \\
(\mathrm{mmol} / \mathrm{L})\end{array}$ & $\begin{array}{l}-0.04 \\
(-0.26 \\
0.18)\end{array}$ & $\begin{array}{l}-0.11 \\
(-0.43 \\
0.2)\end{array}$ & $\begin{array}{l}-0.24 \\
(-0.66 \\
0.18)\end{array}$ & $\begin{array}{l}0.33 \\
(0.01 \\
0.65)\end{array}$ & 0.698 & 0.050 & 0.085 \\
\hline$\Delta \mathrm{HbA} 1 \mathrm{c}(\%)$ & $\begin{array}{l}-0.8(-1.6, \\
0.1)\end{array}$ & $\begin{array}{l}-0.3(-1.0 \\
0.4)\end{array}$ & $\begin{array}{l}-0.2(-0.5 \\
0.2)\end{array}$ & $\begin{array}{l}-0.3(-1.0 \\
0.4)\end{array}$ & 0.506 & 0.820 & 0.229 \\
\hline $\begin{array}{l}\Delta \text { Fasting } \\
\text { blood glucose } \\
(\mathrm{mmol} / \mathrm{L})\end{array}$ & $\begin{array}{l}-0.9(-1.6, \\
-0.2)\end{array}$ & $\begin{array}{l}-1.1(-2.0 \text {, } \\
-0.1)\end{array}$ & $\begin{array}{l}-0.6(-1.3 \\
0.2)\end{array}$ & $\begin{array}{l}0.2(-0.8 \\
1.1)\end{array}$ & 0.337 & 0.884 & 0.798 \\
\hline $\begin{array}{l}\Delta \text { Fasting } \\
\text { insulin }(\mu \mathrm{U} / \mathrm{mL})\end{array}$ & $\begin{array}{l}-1.8(-6.1, \\
2.5)\end{array}$ & $\begin{array}{l}1.5(-1.7 \\
4.6)\end{array}$ & $\begin{array}{l}0.5(-3.8 \\
4.8)\end{array}$ & $\begin{array}{l}1.0(-3.0 \\
5.1)\end{array}$ & 0.806 & 0.342 & 0.594 \\
\hline$\triangle \mathrm{HOMA-IR}$ & $\begin{array}{l}-0.9(-2.4, \\
0.5)\end{array}$ & $\begin{array}{l}-0.2(-1.3, \\
0.8)\end{array}$ & $\begin{array}{l}-0.2(-1.6 \\
1.3)\end{array}$ & $\begin{array}{l}0.8(-1.5 \\
3.0)\end{array}$ & 0.446 & 0.274 & 0.751 \\
\hline $\begin{array}{l}\Delta \text { Matsuda } \\
\text { Index }{ }^{\text {a }}\end{array}$ & $\begin{array}{l}0.34 \\
(-0.48 \\
1.16)\end{array}$ & $\begin{array}{l}0.26 \\
(-0.32 \\
0.83)\end{array}$ & $\begin{array}{l}0.31 \\
(-0.55 \\
1.18)\end{array}$ & $\begin{array}{l}-0.38 \\
(-0.99 \\
0.24)\end{array}$ & 0.573 & 0.159 & 0.628 \\
\hline $\begin{array}{l}\Delta \text { Glucose AUC } \\
\text { a }\end{array}$ & $\begin{array}{l}-78.1 \\
(-225.8 \\
69.6)\end{array}$ & $\begin{array}{l}-140.1 \\
(-307.2 \\
27.0)\end{array}$ & $\begin{array}{l}-75.9 \\
(-201.7 \\
49.8)\end{array}$ & $\begin{array}{l}36.1 \\
(-112.0 \\
184.2)\end{array}$ & 0.197 & 0.716 & 0.208 \\
\hline
\end{tabular}

Data are mean ( $95 \%$ confidence interval). $\Delta$, changes from the endpoint to the pre; VEG: vitamin $D_{3}$ combined exercise intervention group; VDG: vitamin $\mathrm{D}_{3}$ intervention group; $E G$ : exercise intervention group; CG: control group; HOMA-IR, homeostasis model assessment of insulin resistance; $\mathrm{HbA1c}$, Glycated hemoglobin. ${ }^{a}, \mathrm{n}=13$ in VDG, and $\mathrm{n}=13$ in CG were included 


\begin{tabular}{|c|c|c|c|c|c|c|c|}
\hline \multirow[t]{2}{*}{ variable } & VEG & VDG & EG & CG & \multicolumn{3}{|l|}{$P$} \\
\hline & $N=15$ & $N=15$ & $N=14$ & $N=15$ & $\begin{array}{l}P_{\text {vitamin }} \\
\text { D }\end{array}$ & $P_{\text {exercise }}$ & $P_{\text {interaction }}$ \\
\hline$\Delta$ Insulin AUC ${ }^{a}$ & $\begin{array}{l}515.8 \\
(-1106.7, \\
2138.2)\end{array}$ & $\begin{array}{l}1018.1 \\
(-128.1 \\
2164.3)\end{array}$ & $\begin{array}{l}1205.9 \\
(-161.9 \\
2249.9)\end{array}$ & $\begin{array}{l}-149.1 \\
(-1879.0, \\
1680.9)\end{array}$ & 0.724 & 0.529 & 0.173 \\
\hline $\begin{array}{l}\Delta \text { Insulinogenic } \\
\text { Index }{ }^{a}\end{array}$ & $\begin{array}{l}-0.01 \\
(-0.09 \\
0.07)\end{array}$ & $\begin{array}{l}0.09 \\
(0.00 \\
0.17)\end{array}$ & $\begin{array}{l}0.09 \\
(-0.05 \\
0.24)\end{array}$ & $\begin{array}{l}-0.02 \\
(-0.11 \\
0.06)\end{array}$ & 0.937 & 0.801 & 0.032 \\
\hline $\begin{array}{l}\Delta \text { Disposition } \\
\text { Index }{ }^{\text {a }}\end{array}$ & $\begin{array}{l}0.03 \\
(-0.25 \\
0.31)\end{array}$ & $\begin{array}{l}0.28 \\
(-0.19 \\
0.74)\end{array}$ & $\begin{array}{l}0.25 \\
(-0.14 \\
0.64)\end{array}$ & $\begin{array}{l}-0.16 \\
(-0.43 \\
0.10)\end{array}$ & 0.552 & 0.553 & 0.051 \\
\hline \multicolumn{8}{|c|}{$\begin{array}{l}\text { Data are mean ( } 95 \% \text { confidence interval). } \Delta \text {, changes from the endpoint to the pre; VEG: vitamin } D_{3} \\
\text { combined exercise intervention group; VDG: vitamin } D_{3} \text { intervention group; EG: exercise intervention } \\
\text { group; CG: control group; HOMA-IR, homeostasis model assessment of insulin resistance; HbA1c, } \\
\text { Glycated hemoglobin. }{ }^{a}, n=13 \text { in VDG, and } n=13 \text { in CG were included }\end{array}$} \\
\hline
\end{tabular}

During the OGTT, the values of glucose and insulin at the five time points were similar between before and after intervention in the CG, while lower glucose and higher insulin levels were found after intervention than before intervention in the VEG, VDG, and EG, although the difference was not statistically significant (Supplemental Fig. 1). No significant exercise and vitamin D interaction or any main effect of exercise or vitamin D on glucose and insulin during OGTT was observed except for glucose at $30 \mathrm{~min}\left(P_{\text {interaction }}=0.052\right.$, data not shown). In the non-exercise training groups, vitamin $D$ supplementation significantly decreased glucose levels $30 \mathrm{~min}$ after OGTT compared with without vitamin D supplementation (VDG: mean $=-1.6,95 \% \mathrm{Cl}=-2.9--0.2$; CG: mean $=0.3,95 \% \mathrm{Cl}=-1.1-1.6$ ).

\section{Body Fat And Bone Mass}

Vitamin D, but not exercise, significantly attenuated the loss of total BMC (vitamin D: mean $=-5.3 .95 \% \mathrm{Cl}$ $=-29.9-19.4$; exercise: mean $=-49.8,95 \% \mathrm{Cl}=-74.9--24.7)$, trunk BMC (vitamin D: mean $=-2.3,95 \% \mathrm{Cl}=$ -24.1-19.5; exercise: mean $=-33.9,95 \% \mathrm{Cl}=-56.1--11.7$ ), and spine BMD (vitamin $\mathrm{D}$ : mean $=0.00,95 \% \mathrm{Cl}$ $=-0.03-0.03$; exercise: mean $=-0.04,95 \% \mathrm{Cl}=-0.07-0.01$ ) measured using DXA (Table 3 and Fig. 3 ). Exercise, but not vitamin D intervention, significantly decreased total body fat $\%$ (mean $=-1.04,95 \% \mathrm{Cl}=$ $-1.69--0.39$ ) and trunk body fat $\%$ (mean $=-1.45,95 \% \mathrm{Cl}=-2.31--0.59)$ (Table 3 and Fig. 4). There were no vitamin $\mathrm{D}$ and exercise interaction for any body fat and bone mass variables (Table 3 ). Weight, $\mathrm{BMI}$, total body fat \%, trunk body fat\%, total BMC, and trunk BMC significantly decreased after intervention compared with before intervention (0 week) in the EG, but not in the other three groups (Table 3). 
Table 3

Mean differences $(95 \% \mathrm{Cl})$ of body composition from the endpoint with each group

\begin{tabular}{|c|c|c|c|c|c|c|c|}
\hline \multirow[t]{2}{*}{ Variable } & \multirow{2}{*}{$\begin{array}{l}\text { VEG } \\
\mathrm{N}=15\end{array}$} & \multirow{2}{*}{$\begin{array}{l}\text { VDG } \\
N=14\end{array}$} & \multirow{2}{*}{$\begin{array}{l}E G \\
N=14\end{array}$} & \multirow{2}{*}{$\begin{array}{l}\text { CG } \\
N=14\end{array}$} & \multicolumn{3}{|l|}{$P$} \\
\hline & & & & & $\begin{array}{l}P_{\text {vitamin }} \\
\text { D }\end{array}$ & $P_{\text {exercise }}$ & $P_{\text {interaction }}$ \\
\hline $\begin{array}{l}\Delta \text { Weight } \\
(\mathrm{kg})^{\text {a }}\end{array}$ & $\begin{array}{l}-0.3(-1.3 \\
0.7)\end{array}$ & $\begin{array}{l}-0.1 \\
1.4)\end{array}(-1.6$ & $\begin{array}{l}-1.1(-1.9, \\
-0.2)\end{array}$ & $\begin{array}{l}-0.3(-1.3, \\
0.8)\end{array}$ & 0.516 & 0.386 & 0.341 \\
\hline $\begin{array}{l}\triangle \mathrm{BMl} \\
\left(\mathrm{kg} / \mathrm{m}^{2}\right)^{\mathrm{a}}\end{array}$ & $\begin{array}{l}0.8(-1.0 \\
2.5)\end{array}$ & $\begin{array}{l}0.0(-0.6 \\
0.6)\end{array}$ & $\begin{array}{l}-0.3(-0.7, \\
-0.0)\end{array}$ & $\begin{array}{l}-0.1(-0.6 \\
0.3)\end{array}$ & 0.245 & 0.522 & 0.211 \\
\hline $\begin{array}{l}\Delta \text { Total } \\
\text { body fat }(\%)\end{array}$ & $\begin{array}{l}-1.0(-2.1 \\
0.2)\end{array}$ & $\begin{array}{l}0.3(-1.0 \\
1.7)\end{array}$ & $\begin{array}{l}-1.1(-2.0, \\
-0.2)\end{array}$ & $\begin{array}{l}-0.4(-1.1, \\
0.4)\end{array}$ & 0.262 & 0.032 & 0.428 \\
\hline $\begin{array}{l}\Delta \text { Total } \\
\text { body } \\
\text { muscle (g) }\end{array}$ & $\begin{array}{l}529(-194 \\
1252)\end{array}$ & $\begin{array}{l}10(-640 \\
661)\end{array}$ & $\begin{array}{l}414(-404 \\
1232)\end{array}$ & $\begin{array}{l}113 \\
(-485 \\
711)\end{array}$ & 0.758 & 0.134 & 0.505 \\
\hline $\begin{array}{l}\Delta \text { Total } \\
\text { BMD } \\
(\mathrm{g} / \mathrm{cm} 2)\end{array}$ & $\begin{array}{l}-0.01 \\
(-0.02 \\
0.01)\end{array}$ & $\begin{array}{l}-0.00 \\
(-0.01 \\
0.00)\end{array}$ & $\begin{array}{l}-0.00 \\
(-0.01,0.01)\end{array}$ & $\begin{array}{l}-0.01 \\
(-0.01 \\
0.01)\end{array}$ & 0.796 & 0.597 & 0.539 \\
\hline $\begin{array}{l}\triangle \text { Total } \\
\text { BMC (g) }\end{array}$ & $-16(-41,9)$ & $\begin{array}{l}9(-37 \\
55)\end{array}$ & $\begin{array}{l}-73(-118 \\
-27)\end{array}$ & $\begin{array}{l}-29(-71 \\
13)\end{array}$ & 0.014 & 0.062 & 0.485 \\
\hline $\begin{array}{l}\Delta \text { Trunk } \\
\text { body fat (\%) }\end{array}$ & $\begin{array}{l}-1.1(-2.6, \\
0.5)\end{array}$ & $\begin{array}{l}0.4(-1.3 \\
2.1)\end{array}$ & $\begin{array}{l}-1.8(-2.9, \\
-0.7)\end{array}$ & $\begin{array}{l}-0.4(-1.5 \\
0.8)\end{array}$ & 0.156 & 0.023 & 0.856 \\
\hline $\begin{array}{l}\Delta \text { Trunk } \\
\text { muscles (g) }\end{array}$ & $\begin{array}{l}43(-682, \\
68)\end{array}$ & $\begin{array}{l}169 \\
(-515 \\
852)\end{array}$ & $\begin{array}{l}426(-41, \\
892)\end{array}$ & $\begin{array}{l}115 \\
(-452 \\
682)\end{array}$ & 0.523 & 0.442 & 0.479 \\
\hline $\begin{array}{l}\triangle \text { Trunk } \\
\text { BMD } \\
\left(\mathrm{g} / \mathrm{cm}^{2}\right)\end{array}$ & $\begin{array}{l}-0.01(-0.01 \\
0.00)\end{array}$ & $\begin{array}{l}-0.00 \\
(-0.01 \\
0.01)\end{array}$ & $\begin{array}{l}-0.03 \\
(-0.08 \\
0.01)\end{array}$ & $\begin{array}{l}0.01 \\
(-0.02 \\
0.04)\end{array}$ & 0.523 & 0.087 & 0.160 \\
\hline $\begin{array}{l}\triangle \text { Trunk } \\
\text { BMC (g) }\end{array}$ & $-16(-38,5)$ & $\begin{array}{l}16(-19 \\
52)\end{array}$ & $-50(-94,-6)$ & $\begin{array}{l}-14(-51 \\
22)\end{array}$ & 0.046 & 0.600 & 0.912 \\
\hline $\begin{array}{l}\triangle \text { Spine } \\
\text { BMD } \\
\left(\mathrm{g} / \mathrm{cm}^{2}\right)\end{array}$ & $\begin{array}{l}0.02(-0.01 \\
0.04)\end{array}$ & $\begin{array}{l}-0.01 \\
(-0.03 \\
0.00)\end{array}$ & $\begin{array}{l}-0.05 \\
(-0.11 \\
0.02)\end{array}$ & $\begin{array}{l}-0.03 \\
(-0.07 \\
0.02)\end{array}$ & 0.046 & 0.826 & 0.234 \\
\hline $\begin{array}{l}\text { Data are me } \\
\text { from the end } \\
\text { exercise gro } \\
\text { mineral cont }\end{array}$ & $\begin{array}{l}\text { (95\% confid } \\
\text { int to the pre } \\
\text { CG: control }\end{array}$ & e interval) & $\begin{array}{l}n=15 \text { in } \mathrm{V} \\
\mathrm{D}_{3} \text { combine } \\
\text { ly mass inde }\end{array}$ & $\begin{array}{l}\text {, and } n=1 \\
\text { xercise gro } \\
\text { BMD bone }\end{array}$ & $\begin{array}{l}\text { n CG we } \\
\text {; VDG: }\end{array}$ & $\begin{array}{l}\text { included } \\
\text { min } D_{3} \\
\text { sity; } B M\end{array}$ & $\begin{array}{l}\text {, changes } \\
\text { oup; EG: } \\
\text { bone }\end{array}$ \\
\hline
\end{tabular}

\section{Physical Activity And Sun Exposure Score Changes}


There were no group differences in the changes (after-before) in weekly physical activity (Mean \pm SD) (VEG, 1500.1 $\pm 4475.3 ;$ VDG, $-310.8 \pm 4888.9 ; E G,-241.9 \pm 4160.1 ; C G, 509.8 \pm 1541.9 ; P=0.754$ ) and sun exposure score (mean $\pm S D)(V E G,-0.13 \pm 11.9 ; V D G, 5.7 \pm 11.4 ; E G, 4.6 \pm 11.4 ; C G, 5.6 \pm 16.6 ; P=0.667)$ (data not shown).

\section{Discussion}

This RCT found that in type 2 diabetes patients, vitamin D combined with exercise intervention had a potentially beneficial effect on glycemic control, although the effect was not statistically significant. Exercise significantly decreased total and trunk body fat percentage, and vitamin $D$ supplementation significantly reduced bone loss.

In addition to the traditional role in maintaining bone health, higher serum 25(OH)D levels have been found to be associated with a low risk of developing type 2 diabetes ${ }^{7-10}$. However, to date, the findings in previous studies are inconsistent ${ }^{12-17}$, and these studies have mainly been conducted in non-Asian populations. Additionally, accumulating evidence indicates that vitamin D supplementation combined with exercise intervention may have a synergistic effect on the improvement of type 2 diabetes via different glucose utilization pathways ${ }^{21-24}$. However, few studies have investigated the synergistic effect of vitamin $D$ supplementation and exercise training intervention on metabolic profiles in type 2 diabetes patients.

Glycemic control is an important metabolic aspect in the improvement and control of type 2 diabetes and has been identified as a risk factor for the development of diabetic complications ${ }^{32}$. Although there were no vitamin $D$ and exercise main effects or combined effects on fasting blood glucose, insulin, or $\mathrm{HbA} 1 \mathrm{c}$ levels or HOMA-IR, a reduction in glucose levels in the VEG and VDG and an increase in the insulinogenic index in the VDG were observed in this study. However, the effects were not statistically significant, probably due to the relatively small sample size and the relatively short follow-up period. Our results are consistent with those in a study conducted in 42 type 2 diabetes elderly women ${ }^{33}$ but inconsistent with those in a study conducted in type 2 diabetes model rats, in which rats were fed with alfacalcidol instead of vitamin $D^{34}$. Further studies with larger samples and prolonged follow-up periods are warranted to corroborate the current findings.

Exercise is recommended for both prevention and treatment of type 2 diabetes ${ }^{20}$. Consistent with previous findings ${ }^{35}$, we found the main effects of exercise on the reduction of body fat and blood triglyceride, but not on glucose profiles. In addition, previous studies showed that improved glucose profile, for example $\mathrm{HbA} 1 \mathrm{c}$ levels, were not achieved by aerobic or exercise training alone but were observed in the combination of aerobic and resistance training in type 2 diabetes patients ${ }^{36,37}$. In the present study, exercise training was mainly aerobic training, which probably led to a limited effect on glucose profiles. Moreover, HbA1c is a measure of how well blood glucose has been controlled over a 
period of about 3 months ${ }^{38}$ thus, long-term interventions may be needed to improve $\mathrm{HbA} 1 \mathrm{c}$ levels in type 2 diabetes patients with non-insulin dependence.

Weight loss during type 2 diabetes management may lead to a high rate of bone loss, which would increase the risk of future fractures in type 2 diabetes patients ${ }^{4,39}$. In the present study, body weight, BMI, and body fat\%, in addition to total and trunk BMC, were significantly decreased in the exercise alone group, but those results were not observed in the other three groups, suggesting that exercise training alone may increase fracture risk in type 2 diabetes patients accompanied with weight loss. Vitamin $D$ is well known for its beneficial effect in maintaining bone health and reducing the risk of osteoporosis ${ }^{5}$. Consistently, we found that vitamin D supplementation could efficiently maintain total BMC, trunk BMC, and spine BMD. Thus, a combination of vitamin $D$ and exercise intervention in type 2 diabetes management may help mitigate the adverse effects of exercise training on bone health.

China is facing a rising epidemic of NCDs, with no sign of abating. Prevention of NCDs, including diabetes, by promoting healthy eating and lifestyles has become a national public policy priority 2,40 . In October 2016, China issued the "Healthy China 2030," thereby bringing the NCDs issue into a sharper and more concrete focus ${ }^{41}$. The findings in this study of vitamin $D$ supplementation and exercise training in Chinese type 2 diabetes patients could provide a comprehensive understanding of the development of therapeutic strategies for type 2 diabetes.

The strengths of this study include the $2 \times 2$ factorial RCT design, high participant retention and adherence to the intervention, and objective and high-accuracy assessment of glycemic control, body fat, and bone mass. Our study provides a novel finding that combined vitamin $\mathrm{D}$ and exercise training could not only mitigate potential adverse effects during type 2 diabetes management but also have possible benefits in glycemic control. Some limitations of this study should be noted. First, the study duration was relatively short, although it has been demonstrated that body fat and blood metabolic profile changes can be detected during 12 weeks ${ }^{42}$. Second, this study did not comprehensively assess dietary intake, although participants were instructed not to alter their diet; these factors could have affected the results. Thus, future studies should attempt to thoroughly control the diet. Third, the intensity and frequency of exercise training in our study were supervised, and the sample size was relatively small. However, the prepower analysis showed that the sample size in our study would provide a more than $85 \%$ chance to demonstrate the effect.

\section{Conclusions}

To the best of our knowledge, no study has investigated the combined effects of vitamin D supplementation and exercise training on glycemic control and bone health in type 2 diabetes patients. We found that 12 weeks of vitamin $D$ supplementation and exercise training had a potentially positive effect on glycemic control. Circulating 25(OH)D levels substantially increased after vitamin D supplementation, and this increase might play an important role in maintaining the bone mass, which was reduced in the exercise alone group. The present findings shed light on the potential applications of 
vitamin D supplementation combined with exercise training in type 2 diabetes patients. Further studies are required to investigate the long-term effect of a combined vitamin $D$ and exercise intervention on glycemic control and explore its potential mechanisms in type 2 diabetes patients.

\section{Declarations}

\section{Ethics approval and consent to participate}

The purpose, procedures, and risks of the study were explained to each participant before inclusion, and all participants provided written informed consent. All of the procedures were reviewed and approved by the Ethics Committee of Xi'an Jiaotong University Health Science Center. The study was conducted in accordance with the Declaration of Helsinki and registered in the Chinese Clinical Trial System (No. ChiCTR1800015383).

\section{Consent for publication}

Not applicable.

\section{Availability of data and materials}

The data are not publicly available due to research ethical reasons and the corresponding author can provide further information on a reasonable request.

\section{Competing interests}

The authors have no conflicts of interest to declare.

\section{Funding}

This study was supported by the National Natural Science Foundation of China [no. 81703220] and the Fundamental Research Funds for the Central Universities [no. xjj2018148].

\section{Authors' contributions}

X.S. drafted the manuscript. M.D., X. H., Y. M. contributed to data acquisition and/or analysis. S.Z. and W.X. contributed to the interpretation of data. X.S., Y.W., and W.C. contributed to the conception and design of the study. All authors critically revised the manuscript and gave final approval. Y. W. and W. C. are the guarantor of this work and, as such, had full access to all the data in the study and takes responsibility of the data and the accuracy of the data analysis.

\section{Acknowledgements}

We are thankful to all the participants who dedicated their time and effort to complete this study. The authors would like to thank Yixuan Li (Xi'an Jiaotong University Health Science Center), who assisted with randomization and allocation procedures; Ying Pang (First Affiliated Hospital of Xi'an Jiaotong 
University), Sijia Fei (First Affiliated Hospital of Xi'an Jiaotong University), and Lijing Yan (Second Affiliated Hospital of Xi'an Jiaotong University) for helping collect data. Who would like to equally acknowledge the contributions of the technicians at the Department of Health Science of Xi'an Physical Education University.

\section{References}

1. Mortality GBD, Causes of Death C. Global, regional, and national age-sex specific all-cause and cause-specific mortality for 240 causes of death, 1990-2013: A systematic analysis for the global burden of disease study 2013. Lancet. 2015;385:117-71.

2. Wang $L, G a o P$, Zhang $M$, et al. Prevalence and ethnic pattern of diabetes and prediabetes in china in 2013. JAMA. 2017;317:2515-23.

3. Yang W, Lu J, Weng J, et al. Prevalence of diabetes among men and women in china. N Engl J Med. 2010;362:1090-101.

4. Lipkin EW, Schwartz AV, Anderson AM, et al. The look ahead trial: Bone loss at 4-year follow-up in type 2 diabetes. Diabetes Care. 2014;37:2822-9.

5. Holick MF. Vitamin d deficiency. The New England journal of medicine 2007, 357:266-81.

6. Mitri J, Pittas AG. Vitamin d and diabetes. Endocrinol Metab Clin North Am. 2014;43:205-32.

7. Lucato P, Solmi M, Maggi S, et al. Low vitamin d levels increase the risk of type 2 diabetes in older adults: A systematic review and meta-analysis. Maturitas. 2017;100:8-15.

8. Pittas AG, Chung M, Trikalinos $T$, et al. Systematic review: Vitamin $d$ and cardiometabolic outcomes. Ann Intern Med. 2010;152:307-14.

9. Barbarawi M, Zayed Y, Barbarawi O, et al.: Effect of vitamin d supplementation on the incidence of diabetes mellitus. The Journal of clinical endocrinology and metabolism 2020, 105.

10. Pittas AG, Jorde R, Kawahara T, et al.: Vitamin d supplementation for prevention of type 2 diabetes mellitus. To d or not to d? The Journal of clinical endocrinology and metabolism 2020.

11. Zhang J, Ye J, Guo G, et al.: Vitamin d status is negatively correlated with insulin resistance in chinese type 2 diabetes. International journal of endocrinology 2016, 2016:1794894.

12. Krul-Poel $\mathrm{YH}$, Westra $\mathrm{S}$, ten Boekel $\mathrm{E}$, et al. Effect of vitamin d supplementation on glycemic control in patients with type 2 diabetes (sunny trial): A randomized placebo-controlled trial. Diabetes Care. 2015;38:1420-6.

13. Strobel F, Reusch J, Penna-Martinez M, et al.: Effect of a randomised controlled vitamin d trial on insulin resistance and glucose metabolism in patients with type 2 diabetes mellitus. Hormone and metabolic research $=$ Hormon- und Stoffwechselforschung $=$ Hormones et metabolisme 2014, 46:54 -8 .

14. Jafari T, Faghihimani E, Feizi A, et al. Effects of vitamin d-fortified low fat yogurt on glycemic status, anthropometric indexes, inflammation, and bone turnover in diabetic postmenopausal women: A randomised controlled clinical trial. Clin Nutr. 2016;35:67-76. 
15. Tabesh M, Azadbakht L, Faghihimani E, et al. Effects of calcium-vitamin d co-supplementation on metabolic profiles in vitamin d insufficient people with type 2 diabetes: A randomised controlled clinical trial. Diabetologia. 2014;57:2038-47.

16. Elkassaby S, Harrison LC, Mazzitelli N, et al. A randomised controlled trial of high dose vitamin d in recent-onset type 2 diabetes. Diabetes Res Clin Pract. 2014;106:576-82.

17. Parekh D, Sarathi V, Shivane VK, et al. Pilot study to evaluate the effect of short-term improvement in vitamin $d$ status on glucose tolerance in patients with type 2 diabetes mellitus. Endocrine practice: official journal of the American College of Endocrinology the American Association of Clinical Endocrinologists. 2010;16:600-8.

18. Krul-Poel YH, Ter Wee MM, Lips P, et al. Management of endocrine disease: The effect of vitamin d supplementation on glycaemic control in patients with type 2 diabetes mellitus: A systematic review and meta-analysis. Eur J Endocrinol. 2017;176:R1-14.

19. Yiu YF, Yiu KH, Siu CW, et al. Randomized controlled trial of vitamin d supplement on endothelial function in patients with type 2 diabetes. Atherosclerosis. 2013;227:140-6.

20. Venables MC, Jeukendrup AE. Physical inactivity and obesity: Links with insulin resistance and type 2 diabetes mellitus. Diab/Metab Res Rev. 2009;25(Suppl 1):18-23.

21. Aly YE, Abdou AS, Rashad MM, et al. Effect of exercise on serum vitamin $d$ and tissue vitamin $d$ receptors in experimentally induced type 2 diabetes mellitus. Journal of advanced research. 2016;7:671-9.

22. Sun $X$, Cao ZB, Taniguchi H, et al. Effect of an acute bout of endurance exercise on serum 25 (oh)d concentrations in young adults. J Clin Endocrinol Metab. 2017;102:3937-44.

23. Makanae $Y$, Ogasawara $R$, Sato $K$, et al. Acute bout of resistance exercise increases vitamin $d$ receptor protein expression in rat skeletal muscle. Exp Physiol. 2015;100:1168-76.

24. Manna P, Achari AE, Jain SK. Vitamin d supplementation inhibits oxidative stress and upregulate sirt1/ampk/glut4 cascade in high glucose-treated 3t3l1 adipocytes and in adipose tissue of high fat diet-fed diabetic mice. Arch Biochem Biophys. 2017;615:22-34.

25. Weng J, Ji L, Jia W, et al. Standards of care for type 2 diabetes in china. Diabetes Metab Res Rev. 2016;32:442-58.

26. Matsuda M, DeFronzo RA. Insulin sensitivity indices obtained from oral glucose tolerance testing: Comparison with the euglycemic insulin clamp. Diabetes Care. 1999;22:1462-70.

27. Andreozzi F, Mannino GC, Mancuso E, et al. Plasma kisspeptin levels are associated with insulin secretion in nondiabetic individuals. PloS one. 2017;12:e0179834.

28. Hanwell HE, Vieth R, Cole DE, et al. Sun exposure questionnaire predicts circulating 25hydroxyvitamin d concentrations in caucasian hospital workers in southern italy. J Steroid Biochem Mol Biol. 2010;121:334-7.

29. Craig CL, Marshall AL, Sjostrom M, et al. International physical activity questionnaire: 12-country reliability and validity. Med Sci sports Exerc. 2003;35:1381-95. 
30. Sun X, Cao ZB, Tanisawa K, et al. Vitamin d supplementation reduces insulin resistance in japanese adults: A secondary analysis of a double-blind, randomized, placebo-controlled trial. Nutrition research. 2016;36:1121-9.

31. Faul F, Erdfelder E, Lang AG, et al. G*power 3: A flexible statistical power analysis program for the social, behavioral, and biomedical sciences. Behav Res Methods. 2007;39:175-91.

32. Woerle HJ, Neumann C, Zschau S, et al. Impact of fasting and postprandial glycemia on overall glycemic control in type 2 diabetes importance of postprandial glycemia to achieve target hba1c levels. Diabetes Res Clin Pract. 2007;77:280-5.

33. Kim HJ, Kang CK, Park H, et al. Effects of vitamin d supplementation and circuit training on indices of obesity and insulin resistance in $\mathrm{t} 2 \mathrm{~d}$ and vitamin $\mathrm{d}$ deficient elderly women. $\mathrm{J}$ Exerc Nutrition Biochem. 2014;18:249-57.

34. Akagawa M, Miyakoshi N, Kasukawa Y, et al. Effects of activated vitamin d, alfacalcidol, and lowintensity aerobic exercise on osteopenia and muscle atrophy in type 2 diabetes mellitus model rats. PloS one. 2018;13:e0204857.

35. Kim KB, Kim K, Kim C, et al. Effects of exercise on the body composition and lipid profile of individuals with obesity: A systematic review and meta-analysis. J Obes Metab Syndr. 2019;28:27894.

36. Church TS, Blair SN, Cocreham S, et al. Effects of aerobic and resistance training on hemoglobin a1c levels in patients with type 2 diabetes: A randomized controlled trial. Jama. 2010;304:2253-62.

37. Pan $B, G e L, X u n Y Q$, et al. Exercise training modalities in patients with type 2 diabetes mellitus: $A$ systematic review and network meta-analysis. Int J Behav Nutr Phys Act. 2018;15:72.

38. Tahara Y, Shima K. Kinetics of hba1c, glycated albumin, and fructosamine and analysis of their weight functions against preceding plasma glucose level. Diabetes Care. 1995;18:440-7.

39. Daly RM, Dunstan DW, Owen N, et al. Does high-intensity resistance training maintain bone mass during moderate weight loss in older overweight adults with type 2 diabetes? Osteoporosis Int. 2005;16:1703-12.

40. Zimmet PZ, Magliano DJ, Herman WH, et al. Diabetes: A 21st century challenge. The lancet Diabetes endocrinology. 2014;2:56-64.

41. Tan X, Wu Q, Shao H. Global commitments and china's endeavors to promote health and achieve sustainable development goals. J Health Popul Nutr. 2018;37:8.

42. Cao $L$, Jiang $Y, L i ~ Q$, et al. Exercise training at maximal fat oxidation intensity for overweight or obese older women: A randomized study. J Sports Sci Med. 2019;18:413-8.

\section{Figures}




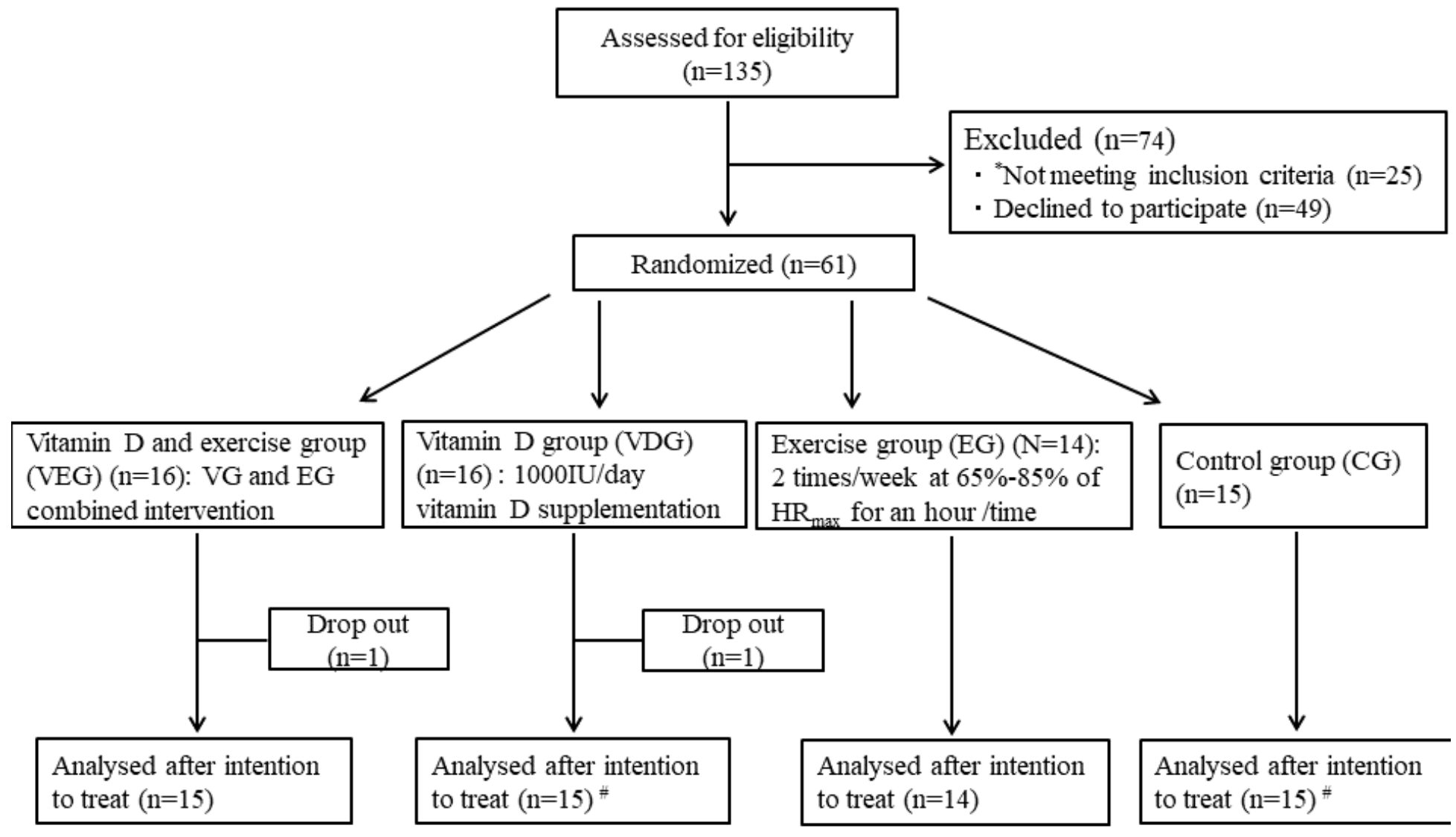

Figure 1

Flow diagram of participants *Most patients did not meet the inclusion criteria because of insulin therapy. \#one participant in each VDG and CG for DXA measurement, and two participants in each VDG and CG for the OGTT did not complete. MHR, maximum heart rate.

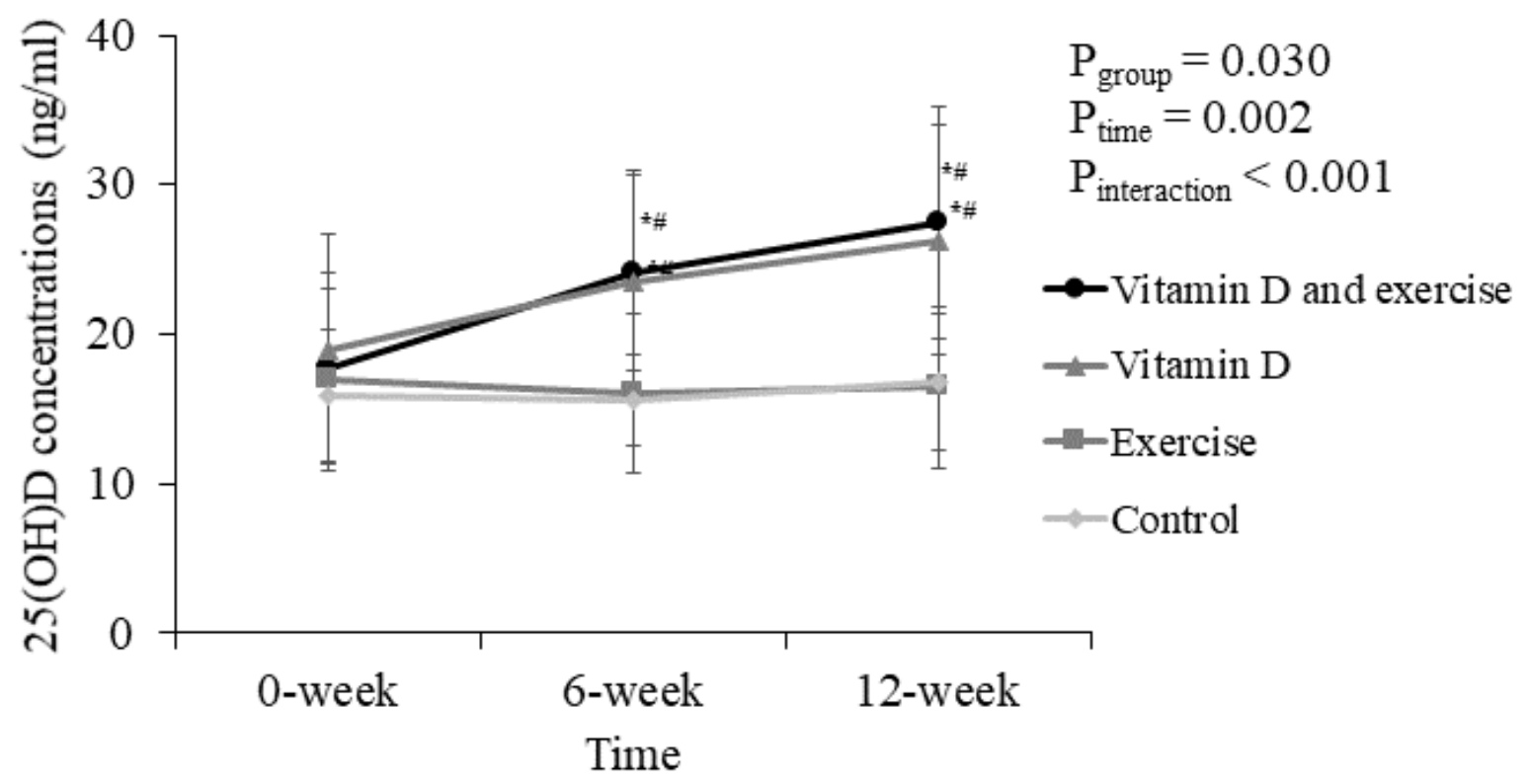


Figure 2

Effect of vitamin D supplementation and exercise training on serum 25(OH)D concentrations in 59 type 2 diabetes Values are expressed as means \pm standard deviation. Two way repeated-measures ANOVA was used to determine the effect of vitamin $D$ and exercise on the serum $25(\mathrm{OH}) \mathrm{D}$ changes. ${ }^{*}, \mathrm{P}<0.05$ vs the baseline within group; \#, $\mathrm{P}<0.05$ vs exercise or control group at the same time point.
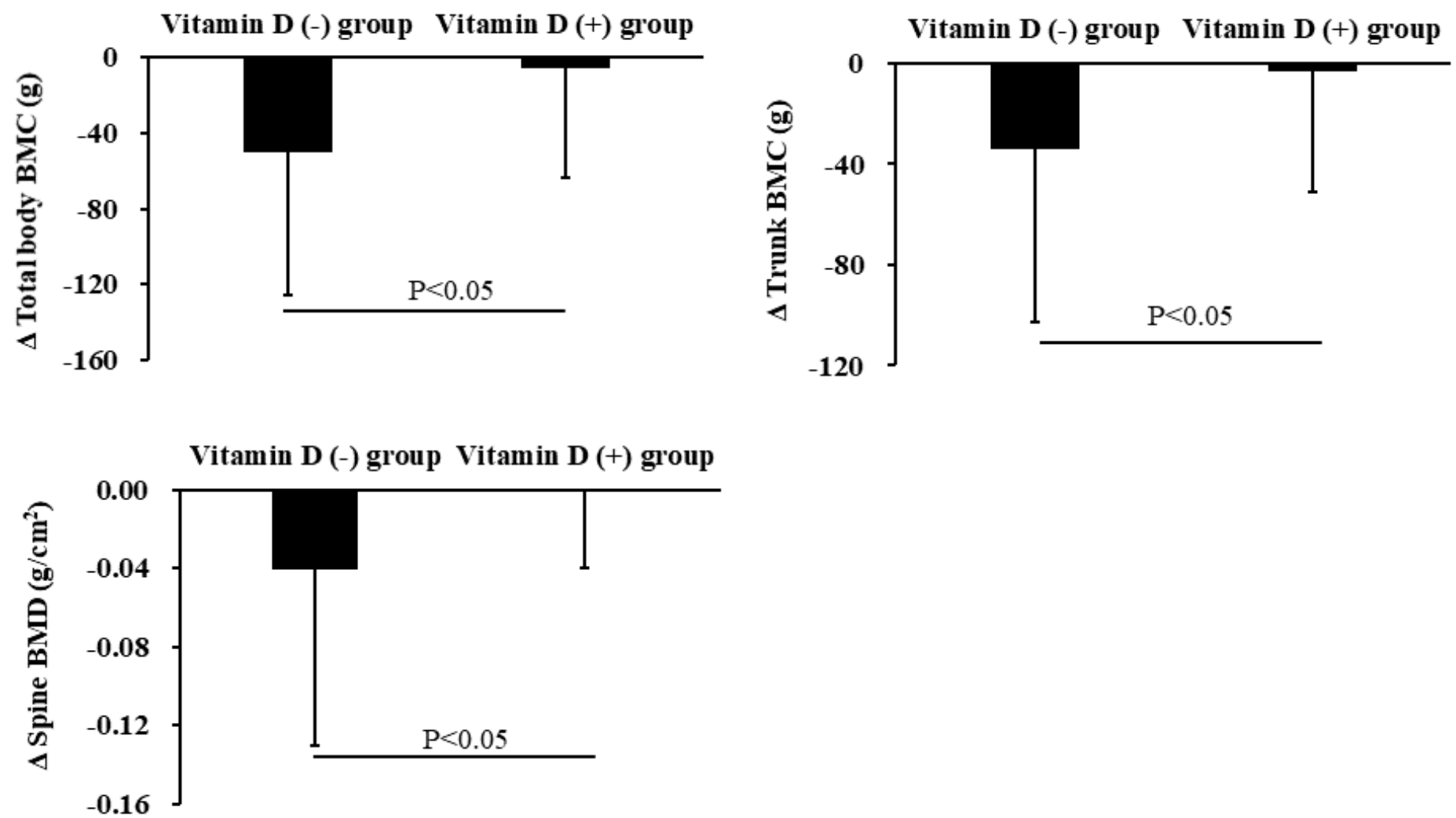

\section{Figure 3}

Effect of vitamin D supplementation on total body BMC (a), trunk BMC (b), and Spine BMD according to vitamin $D$ treatment group* Values are expressed as mean changes \pm standard deviation from the baseline. *vitamin $D(-), n=28$; vitamin $D(+), n=29$. BMC, bone mineral content; $B M D$, bone mineral density. 

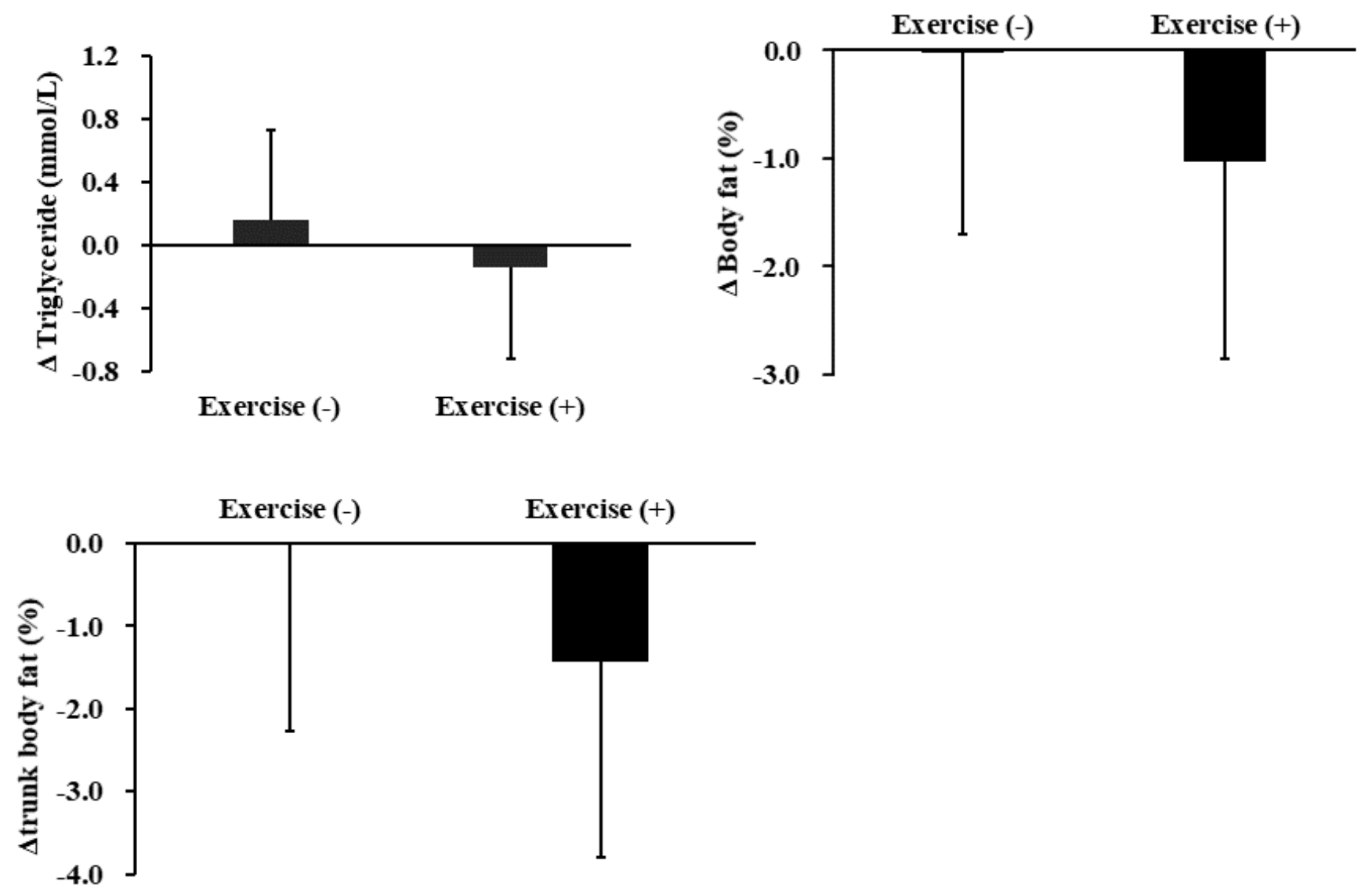

\section{Figure 4}

Effect of exercise training on triglyceride (a), body fat\% (b) and trunk body fat \% (c) according to exercise intervention group ${ }^{*}$ Values are expressed as mean changes \pm standard deviation from the baseline. *vitamin $D(-), n=28$; vitamin $D(+), n=29$.

\section{Supplementary Files}

This is a list of supplementary files associated with this preprint. Click to download.

- SuppFigures.pptx 